\section{Histologische Typisierung beim Adenokarzinom}

In der neuestes WHO-Klassifikation der Adenokarzinome der Lunge spiegelt sich deren Heterogenität in Form einer Subtypisierung der prädominanten histologischen Muster wider. Nun wurde die prädiktive Aussagekraft geprüft.

D ie aktuelle Klassifikation invasiver Adenokarzinome der Lunge umfasst lepidische (LEP), papilläre (PAP), azinöse (ACN), mikropapilläre (MIP) und solide Wachstumsmuster (SOL). Der prädiktive Wert dieser Subtypisierung mit Blick auf das Überleben nach adjuvanter Chemotherapie war bislang nicht in gröBeren Studien untersucht worden. Von 1.766 Patienten mit nichtkleinzelligem Lungenkarzinom (NSCLC) in der LACE-Bio-Studie hatten 725 ein Adenokarzinom, von denen 582 in das neue System klassifiziert werden konnten: LEP $(\mathrm{n}=24), \operatorname{ACN}(\mathrm{n}=152), \operatorname{PAP}(\mathrm{n}=$ 99), MIP ( $n=40)$ und SOL $(n=267)$. Für die Analyse wurden daraus drei Gruppen gebildet: LET, ACN/PAP und MIP/
SOL. Primärer Endpunkt war das Gesamt-, sekundäre Endpunkte waren krankheitsfreies und spezifisches krankheitsfreies Überleben. Bezüglich des Gesamtüberlebens unterschieden sich die histologischen Subgruppen nicht. Jedoch waren in der univariaten Analyse krankheitsfreies und spezifisches krankheitsfreies Überleben in der Subgruppe MIP/SOL schlechter als in den Subgruppen LEP und ANC/PAP $(p<0,01)$.

Von der prädiktiven Analyse zur adjuvanten Chemotherapie wurde der LEPSubtyp wegen zu geringer Zahlen ausgeschlossen. Die Analyse beruhte daher auf den Daten von 552 Patienten, von denen 280 beobachtet und 272 adjuvant behandelt worden waren.
MIP/SOL-Patienten profitierten beim krankheitsfreien (Hazard Ratio [HR] $0,60$; Interaktion $p<0,01)$ und spezifischen krankheitsfreien Überleben (HR 0,59 ; Interaktion $\mathrm{p}=0,01$ ) signifikant von der adjuvanten Chemotherapie, beim Gesamtüberleben nur tendenziell (HR 0,71; Interaktion $\mathrm{p}=0,18$ ).

Patienten der ACN/PAP-Subgruppe hatten durch die adjuvante Chemotherapie keine Vorteile in einer der drei Überlebenskategorien.

Fazit: Die neue Klassifikation des Adenokarzinoms nach prädominanten histologischen Wachstumsmustern ist für das zu erwartende krankheitsfreie und spezifische krankheitsfreie Überleben nach adjuvanter Chemotherapie aussagekräftig, nicht jedoch für das Gesamtüberleben.

Kathrin von Kieseritzky

Tsao M.-S. et al. Subtype Classification of Lung Adenocarcinoma Predicts Benefit From Adjuvant Chemotherapy in Patients Undergoing Complete Resection. J Clin Oncol. 2015;33(30): 3439-46.

\title{
Rolle der stereotaktischen Bestrahlung bei NSCLC bestätigt
}

\author{
Beim frühen nichtkleinzelligen \\ Lungenkarzinom (NSCLC) ist die \\ Chirurgie die Therapie der Wahl. Ist \\ sie nicht möglich, etwa bei betagten \\ Patienten und solchen mit vielen \\ Komorbiditäten, kommt die stereo- \\ taktische Bestrahlung (SBRT) infrage.
}

$\mathrm{D}$ ie Auswertung von Daten aus der SEER(Surveillance, Epidemiology, and End Results)-Datenbank des Medicare-Versicherungssystem in den USA bestätigt dieses Vorgehen. James B. Yu und Kollegen identifizierten Patienten in einem Alter von 67 Jahren und darüber, die zwischen 2007 und 2009 wegen eines NSCLC des Stadiums I operiert $(\mathrm{n}=711)$ oder stereotaktisch bestrahlt $(\mathrm{n}=367)$ worden waren. In ihrer Auswertung verglichen die Wissenschaftler die Patienten aus den beiden Therapie-Gruppen auch in Abhängigkeit von einer kurzen $(<5$ Jahre) oder einer längeren Lebenserwartung ( $\geq 5$ Jahre).
Für die Gesamtgruppen ergaben sich auf kurze Sicht Vorteile für die SBRT: Die akute Toxizität (bis zu einem Monat) war ebenso signifikant geringer (7,9 vs. $54,9 \%, \mathrm{p}<0,001)$ wie die Gesamtmortalität über drei Monate (2,2 vs. $6,1 \%, p=$ 0,005). Das hatte sich nach 24 Monaten relativiert: Zu diesem Zeitpunkt zeigten die Toxizitätsraten keine Unterschiede mehr (69,7 vs. $73,9 \%, p=0,31)$. Bei SBRT traten 647,5 Komplikationen pro 1.000 Personenjahre auf, bei chirurgischer Therapie $1.025,4$ pro 1.000 Personenjahre (Inzidenzratenverhältnis [IRR] 0,74; 95\%-Konfidenzintervall [95\%-KI] 0,640,87 . Die Gesamtmortalität zeigte nun sogar eine höhere Rate nach SBRT im Vergleich zur chirurgischen Therapie ( 40,1 vs. $22,3 \%$; $p<0,001$ ). Bei kurzer Lebenserwartung von bis zu fünf Jahren war dabei die lungenkrebsspezifische Mortalität in beiden Therapiegruppen vergleichbar (IRR 1,01; 95\%-KI 0,40$2,56)$. Anders bei Patienten mit längerer Lebenserwartung, die sowohl im Hin- blick auf die Gesamtmortalität als auch auf die lungenkrebsspezifische Mortalität von der Operation profitierten. Für die Gesamtmortalität lag die IRR von SBRT zu Chirurgie bei 1,49 (95\%-KI $1,11-2,01)$, für die lungenkrebsspezifische Mortalität bei 1,63 (95\%-KI 0,952,79).

Fazit: Die SBRT war assoziiert mit einer geringeren akuten Mortalität und Toxizität, bei Patienten mit längerer Lebenserwartung verschwand dieser Vorteil aufgrund verzögert auftretender Komplikationen und einer längerfristig höheren Mortalität bei SBRT wieder. Das bestätigt den Einsatz der SBRT vorrangig bei Patienten, die für eine Chirurgie nicht infrage kommen oder die eine aufgrund von Alter und Komorbiditäten kurze Lebenserwartung haben.

Friederike Klein

Yu JB et al. Comparative Effectiveness of Surgery and Radiosurgery for Stage I Non-Small Cell Lung Cancer. Cancer. 2015;121(14):2341-9. 